\title{
Urinary Neutrophil Gelatinase Associated Lipocalin as a Marker of Tubular Damage in Type 2 Diabetic Patients with and without Albuminuria
}

\author{
Abeer A. Al-Refai', Safaa I. Tayel'1, Ahmed Ragheb², Ashraf G. Dala², Ahmed Zahran²* \\ ${ }^{1}$ Biochemistry Department, Faculty of Medicine, Menofia University, Shibin El Kom, Egypt \\ ${ }^{2}$ Internal Medicine Department, Faculty of Medicine, Menofia University, Shibin El Kom, Egypt \\ Email: ${ }^{*}$ ahmed173@hotmail.com
}

Received 29 December 2013; revised 28 January 2014; accepted 26 February 2014

Copyright (C) 2014 by authors and Scientific Research Publishing Inc.

This work is licensed under the Creative Commons Attribution International License (CC BY). http://creativecommons.org/licenses/by/4.0/

\section{Open Access}

\section{Abstract}

Background: Neuttrophil gelatinase associated lipocalin (NGAL) was shown to be a good marker for predicting acute kidney injury (AKI). Some recent reports demonstrated that NGAL may be an early biomarker for kidney affection in diabetic patients. The aim of this work is to investigate urinary NGAL (UNGAL) in type 2 diabetic patients with and without albuminuria. Methods: This study included 46 type 2 diabetic patients and 15 healthy age and sex matched individuals as the control group. Diabetic patients were divided into three groups according to urinary albumin excretion (UAE), normoalbuminuria, microalbuminuria and macroalbuminuria. UNGAL was measured in all populations and corrected to urinary creatinine to account for day to day variation in urine volume and transformed log. Comparison between 4 groups (control, normoalbuminuria, microalbuminuria and macroalbuminuria) was done. Results: Log UNGAL/Creatinine ratio showed significant difference when comparing control group $(0.70 \pm 0.58)$ versus normoalbuminuria $(1.71 \pm 1.06)$, microalbuminuria $(1.57 \pm 0.72)$ and macroalbuminuria $(1.92 \pm 0.63)$, however, there was no significant difference among diabetic groups. Pearson's correlation showed that $\log$ UNGAL/Creatinine ratio positively correlated with glycated hemoglobin (HbA1c) and inversely with estimated glomerular filtration rate (eGFR). Regression analysis showed that HbA1c, urinary creatinine and eGFR were the independent predictors of log UNGAL/Creatinine ratio. Conclusion: Tubular markers like UNGAL may be early elevated in type 2 diabetic patients even before the incidence of glomerular injury detected by microalbuminuria and it can be used as an early marker for detection of kidney involvement in diabetic patients.

${ }^{*}$ Corresponding author.

How to cite this paper: Al-Refai, A.A., Tayel, S.I., Ragheb, A., Dala, A.G. and Zahran, A. (2014) Urinary Neutrophil Gelatinase Associated Lipocalin as a Marker of Tubular Damage in Type 2 Diabetic Patients with and without Albuminuria. Open Journal of Nephrology, 4, 37-46. http://dx.doi.org/10.4236/ojneph.2014.41006 


\section{Keywords}

\section{Neutrophil Gelatinase Associated Lipocalin (NGAL); Diabetic Nephropathy; Albuminuria; Tubular Markers}

\section{Introduction}

Diabetes mellitus is the leading cause of chronic kidney disease (CKD), and the rapidly increasing prevalence of diabetes worldwide virtually assures that the proportion of CKD attributable to diabetes will continue to rise [1]. Diabetic kidney disease is associated with enhanced morbidity and mortality, particularly with accelerated cardiovascular disease [2]. The earliest clinical evidence of nephropathy is elevated urine albumin level $\geq 30 \mathrm{mg} /$ 24hours [3]. Microalbuminuria is generally considered as the earliest non-invasive marker for the development of diabetic nephropathy [4], however, microalbuminuria is diagnosed once significant glomerular damage has occurred [5]. Although glomerular dysfunction is thought to be a major factor for the development and progression of diabetic nephropathy, tubulointerstitial damage might also play an important role in the pathogenesis of diabetic nephropathy [6]. It has been suggested that an early increase in urinary albumin excretion is relevant to proximal tubular damage or dysfunction in addition to glomerular permeability [7]. Several different markers of tubular damage have been widely investigated in the areas related to acute kidney injury (AKI) and have presented promising data as early predictive biomarkers of AKI [8] [9]. Recently, some investigators have demonstrated that these tubular markers have clinical importance as biomarkers of diabetic nephropathy [10] [11]. NGAL is an ubiquitous protein of 178 amino acids with a molecular mass of approximately $25 \mathrm{kDa}$. It belongs to the family of lipocalins, which is a family of proteins that have been associated with many biological processes, such as inflammation, the transport of pheromones and the synthesis of prostaglandins [12] [13]. NGAL was initially identified by Allen and Venge in 1989 from human neutrophils, and it is now known that it is expressed at low levels in several human tissues, including kidney, trachea, lungs, stomach and colon [14], However, its precise role has been clarified by Paragas et al. who found that the timing and the intensity of NGAL mRNA and protein were correlated and dependent on the degree of kidney damage in an experimental model of mouse with induced AKI and they found that its production was located in the distal convoluted tubule and the collecting duct while the proximal tubule was involved in the process of NGAL reabsorption [15]. NGAL is produced and secreted into the urine in response to ischemic kidney damage and is therefore a promising early indicator of tubulointerstitial damage [16]. Several recent reports showed that NGAL is an important biomarker and is elevated in DKD [10] [11] [17]. The aim of this work is to evaluate the UNGAL in patients with type 2 diabetes with and without albuminuria.

\section{Patient and Methods}

This study included 46 type 2 diabetic patients on either oral anti diabetic or insulin therapy. In addition, 15 apparently healthy, non-diabetic, without CKD, age and gender matched subjects were included in this study as a control group. All the patients were recruited from outpatient clinic, Internal Medicine Department, Menofia University hospital. The protocol for this study followed the ethical standards and approved by the ethical committee of our institution and all subjects gave informed consent to participate in this study. All patients fulfilled the following inclusion criteria, Type 2 Diabetes mellitus (DM) more than one year, older than 18 years old with stable kidney function defined as stable serum creatinine with less than $10 \%$ change in last three months. The following patients were excluded, Type $1 \mathrm{DM}$, unstable kidney function or kidney disease other than diabetic nephropathy, pregnancy, malignancy, infections or any inflammatory conditions. Patients were divided according urinary albumin excretion (UAE) which was measured by spot morning urine sample for albumin to creatinine ratio (UACR) into 3 groups diabetic with normoalbuminuria (UACR less than $30 \mathrm{mg} / \mathrm{gm}$ ), diabetic with microalbuminuria (UACR between $30-299 \mathrm{mg} / \mathrm{gm}$ ) and diabetic with macroalbuminuria (UACR equal or more than $300 \mathrm{mg} / \mathrm{gm}$ ). All subjects underwent full history taking and clinical examination, including weight, height and measuring blood pressure. Mean arterial pressure (MAP) was calculated as $\{(2 \times$ diastolic blood pressure $(\mathrm{mmHg})+$ systolic blood pressure $(\mathrm{mmHg})\} / 3$. BMI Was calculated as weight $(\mathrm{Kg}) /\{\text { Height }(\mathrm{m})\}^{2}$ and GFR was estimated using Modification of Diet in Renal Disease Abbreviated Equation (MDRD): [GFR $=186 \times$ (se- 
rum Cr $)^{-1.154} \times(\text { age })^{-0.203} \times(0.742$ if female $) \times(1.210$ if African American $\left.)\right][18]$.

\section{Laboratory Assessment}

Venous blood samples were collected following an overnight fasting for measurements of fasting plasma glucose, glycosylated hemoglobin (HbA1c), creatinine and serum urea. A whole blood was obtained by using EDTA containing tube for measurement of glycosylated hemoglobin (HbA1c). Fasting plasma glucose was determined by enzymatic colorimetric test, using Spinreact kit, SPAIN [19]. Serum and urine creatinine concentration was assessed by a kinetic Jaffe' method [20] and serum urea by enzymetic colorimetry methods [21]. Glycosylated hemoglobin was assayed by quantitative colorimetric measurement of glycohemoglobin as percent of total hemoglobin using kits supplied by Teco Diagnostics, Lakeview Ave, Anaheim, CA, USA [22]. The Urinary albumin concentration was measured by a commercially available solid phase enzyme linked immunosorbent assay kit provided by DRG International Inc., USA; cat no EIA-2361], according to manufacturer s instructions from early morning spot urine collections [23] and adjusted for urinary creatinine which has been found to be an accurate measure of urinary albumin excretion per 24 hours [24]. Urinary NGAL was measured in one morning spot urine sample collected in labeled sterile container and thereafter was immediately centrifuged at $4^{\circ} \mathrm{C}$ for $10 \mathrm{~min}$ at $4000 \mathrm{rpm}$ to obtain supernatant. Supernatant of urine were stored at $-20^{\circ} \mathrm{C}$ till analysis. UrineNGAL was measured using an ELISA method according to manufacturer s instructions and provided by [Ray Biotech, Inc. Norcross, GA; Cat\#: ELH-Lipocalin2-001] and adjusted for urine creatinine to account for day-today variation in urine volume, as 24-h urine samples were not available [25]. It was expressed as Pg/mg.

\section{Statistical Evaluations}

We used the statistical package of social signs (SPSS, version 16) to perform the analysis. Categorical data were presented as number and percentages and continuous variables as means \pm standard deviation (SD). One way ANOVA test or Kruskalwalis test was used as appropriate for comparison of quantitative variables more than two independent groups. Chi-Square test was used for categorical variables and Fischer exact test was used if one cell less than 5. T-test or Mann-Whitney test was used as appropriate for comparison between two quantitative variables. The Kolmogorov-Smirnov test was done to determine the distribution of data. Log transformation of UNGAL/Creatinine ratio was done improve interpretability and data scatter. The correlations between the variables were analyzed using Pearson's correlation. Regression analysis was performed to detect the possible predictor of log UNGAL/Creatinine ratio as a marker of tubular injury. Receiving operation characteristic (ROC) curve was constructed to detect cut off value of log UNGAL/Creatinine ratio at which tubular injury can occur comparing normal group with whole diabetic patients. P value $\leq 0.05$ was considered significant.

\section{Results}

This study included 46 patients with type 2 diabetes mellitus with 15 healthy persons as a control group. The diabetic patients were divided into 3 groups according (UACR). So the whole cohort was divided into 4 groups. Group 1: Control group consists of 15 health persons 11(73.3\%) male and 4( 26.7\%) female; Group 2: Diabetic patients with normoalbuminuria (UACR < $30 \mathrm{mg} / \mathrm{gm})$ consists of 16 patients, $12(75 \%)$ males and $4(25 \%)$ females; Group 3: Diabetic patients with microalbuminuria (UACR between $30-299 \mathrm{mg} / \mathrm{gm}$ ) consists of 14 patients, 8 (57.1\%) males and 6 (42.9\%) females; Group 4: Diabetic patients with macroalbuminuria (UACR > 300 $\mathrm{mg} / \mathrm{gm}$ ) consists of 16 patients, 7 (43.8\%) males and 9 (56.2\%) females. All groups were matched regarding age, sex and BMI. Base line characteristics and comparison between studied groups are shown in Table 1. All groups were compared together using ANOVA or Kruskalwalis test as appropriate then each two groups were compared together using student $t$ test or Mann-Whitney test as appropriate. There was significant difference among the studied groups regarding log UNGAL/Creatinine ratio with $\mathrm{P}$ value 0.0001 . Analysis between each two groups showed that there was a significant difference between group 1 (Control group) and each group separately while the diabetic groups did not show significant difference among themselves (Figure 1). Correlation between log UNGAL/Creatinine ratio with HbA1c, eGFR, Duration of diabetes in years and UACR showed significant positive correlation with $\mathrm{HbA1c}$ with $\mathrm{r}$ value +0.4 and $\mathrm{P}$ value 0.01 and significant negative correlation with eGFR with $r$ value -0.4 and $P$ value 0.02 while it did not show significant correlation with UACR and duration of diabetes mellitus (Figure 2) Regression analysis demonstrated that HbA1c, urinary creatinine and 
Table 1. Demographics and comparison between the studied groups.

\begin{tabular}{|c|c|c|c|c|c|}
\hline Variable & $\begin{array}{c}\text { (Group 1) } \\
\text { Control group } \\
\text { UACR (mg/gm) } \\
4.93 \pm 2.26 \\
(\mathrm{n}=15)\end{array}$ & $\begin{array}{c}\text { (Group 2) } \\
\text { Diabetics with } \\
\text { normoalbuminuria } \\
\text { UACR }(\mathrm{mg} / \mathrm{gm}) \\
6.38 \pm 8.42 \\
(\mathrm{n}=16)\end{array}$ & $\begin{array}{c}\text { (Group 3) } \\
\text { Diabetics with } \\
\text { microalbuminuria } \\
\text { UACR (mg/gm) } \\
\begin{array}{c}113.21+51.83 \\
(\mathrm{n}=14)\end{array}\end{array}$ & $\begin{array}{c}\text { (Group 4) } \\
\text { Diabetics with } \\
\text { macroalbuminuria } \\
\text { UACR }(\mathrm{mg} / \mathrm{gm}) \\
1812.6+2074.5 \\
(\mathrm{n}=16)\end{array}$ & P Value \\
\hline Age in years & $50.67 \pm 5.70$ & $48.63 \pm 16.78$ & $56.21 \pm 11.11$ & $54.94 \pm 7.42$ & $>0.05^{\$}$ \\
\hline Sex (M/F) (No and \%) & $11 / 4(73.3 / 26.7)$ & $12 / 4(75 / 25)$ & 8/6 (57.1/42.9) & $7 / 9(43.8 / 56.2)$ & $>0.05^{\#}$ \\
\hline Duration of DM (years) & - & $7.69 \pm 4.66$ & $10.36 \pm 2.82$ & $15.19 \pm 5.32$ & $\begin{array}{c}0.01^{\$} \\
\left(<0.05^{\# \#,+}\right) \\
\left(>0.05^{\# \#}\right)\end{array}$ \\
\hline $\operatorname{BMI}\left(\mathrm{kg} / \mathrm{m}^{2}\right)$ & $29.2+3.39$ & $27.96 \pm 7.34$ & $28.53 \pm 2.12$ & $28.24 \pm 3.45$ & $>0.05^{\$}$ \\
\hline MAP (mmHg) & $88.00 \pm 6.88$ & $100.21 \pm 11.66$ & $100.36 \pm 10.24$ & $100.52 \pm 5.19$ & $\begin{array}{c}0.001^{\$} \\
\left(<0.05^{*, * * * * *, \# \#}\right) \\
\left(>0.05^{\#,+}\right)\end{array}$ \\
\hline $\begin{array}{l}\text { Fasting blood glucose } \\
\text { (mg/dl) }\end{array}$ & $92.40 \pm 2.47$ & $147.12 \pm 30.13$ & $181.12 \pm 49.83$ & $166.31 \pm 46.74$ & $\begin{array}{c}0.0001^{\$} \\
\left(<0.05^{*, * *, * * *, \#}\right) \\
\left(>0.05^{\# \#,++}\right)\end{array}$ \\
\hline HbA1c \% & $4.88 \pm 0.82$ & $9.22 \pm 3.50$ & $6.82 \pm 1.99$ & $7.76 \pm 3.60$ & $\begin{array}{c}0.001^{\$} \\
\left(<0.05^{*, * *, * * *, \#}\right) \\
\left(>0.05^{\# \#,++}\right)\end{array}$ \\
\hline Creatinine (mg/dl) & $0.7 \pm 0.13$ & $1.58 \pm 0.88$ & $1.98 \pm 1.01$ & $3.94 \pm 1.84$ & $\begin{array}{c}0.0001^{\$} \\
\left(<0.05^{* * * * *, \# \#,+}\right) \\
\left(>0.05^{*, \#}\right)\end{array}$ \\
\hline Urea (mg/dl) & $21.00 \pm 2.20$ & $37.56 \pm 30.99$ & $42.64 \pm 28.89$ & $74.81 \pm 40.49$ & $\begin{array}{c}0.0001^{\$} \\
\left(<0.05^{* * *, \# \#,+}\right) \\
\left(>0.05^{*, * *, \#}\right)\end{array}$ \\
\hline Urinary creatinine (mg/dl) & $100.24 \pm 29.42$ & $76.87 \pm 27.21$ & $79.14 \pm 24.85$ & $75.63 \pm 31.79$ & $\begin{array}{c}0.0001^{\$} \\
\left(<0.05^{*, * * * * * * *}\right) \\
\left(0.05^{\#, \#,+,}\right)\end{array}$ \\
\hline eGFR ( ml/min/1.73m²) & $93.89 \pm 16.79$ & $72.73 \pm 59.84$ & $46.32 \pm 30.26$ & $20.17 \pm 16.01$ & $\begin{array}{c}0.0001^{\$} \\
\left(<0.05^{*, * * * * * *, \ldots \#,+}\right) \\
\left(>0.05^{\#}\right)\end{array}$ \\
\hline $\begin{array}{l}\text { Log UNGAL/Creatinine } \\
\text { ratio (Pg/mg) }\end{array}$ & $0.70 \pm 0.58$ & $1.71 \pm 1.06$ & $1.57 \pm 0.72$ & $1.92 \pm 0.63$ & $\begin{array}{c}0.0001^{\$} \\
\left(<0.05^{*, * * *, * * * *}\right) \\
\left(>0.05^{\#, \ldots \#,+,}\right)\end{array}$ \\
\hline
\end{tabular}

\$: All groups together, ${ }^{*}$ : Control VS Normoalbuminuria, ${ }^{* *}$ : Control VS Microalbuminuria, ${ }^{* * *}:$ Control VS Macroalbuminuria, ${ }^{*}:$ Normoalbuminuria VS Microalbuminuria, ${ }^{\#}$ : Normoalbuminuria VS Macroalbuminuria, ${ }^{+}$: Microalbuminuria VS Macroalbuminuria. ACR: Albumin creatinine ratio, M/F: Male/Female, BMI: Body mass index, MAP: Mean arterial pressure, HbA1c: Glycated hemoglobin, eGFR: estimated glomerular filtration rate, Log U NGAL/Creatinine: Logarithm Urinary neutrophil gelatinase associated lipocalin/Creatinine.

eGFR were the independent predictors of Log UNGAL/Creatinine ratio (Table 2). ROC curve was constructed to detect cut off value of Log UNGAL/Creatinine ratio considering control group as status variable. It showed a best cut off value at $1.035 \mathrm{Pg} / \mathrm{mg}$ with good area under the curve (AUC) of 0.87 and sensitivity of $80.4 \%$ and specificity of $80 \%$ (Figure 3 and Table 3 ).

\section{Discussion}

Diabetic nephropathy is currently the leading cause of CKD. It is also one of the most significant long-term complications in terms of morbidity and mortality for individual patients with diabetes [26]. Serum and urinary NGAL are arguably the most promising emerging biomarkers for early detection of AKI [27]. Several recent studies also have defined the role of NGAL in CKD and showed serum and UNGAL levels are markers of kidney disease and severity in CKD [10] [11] [28] [29]. The aim of this work is to evaluate the UNGAL in type 2 


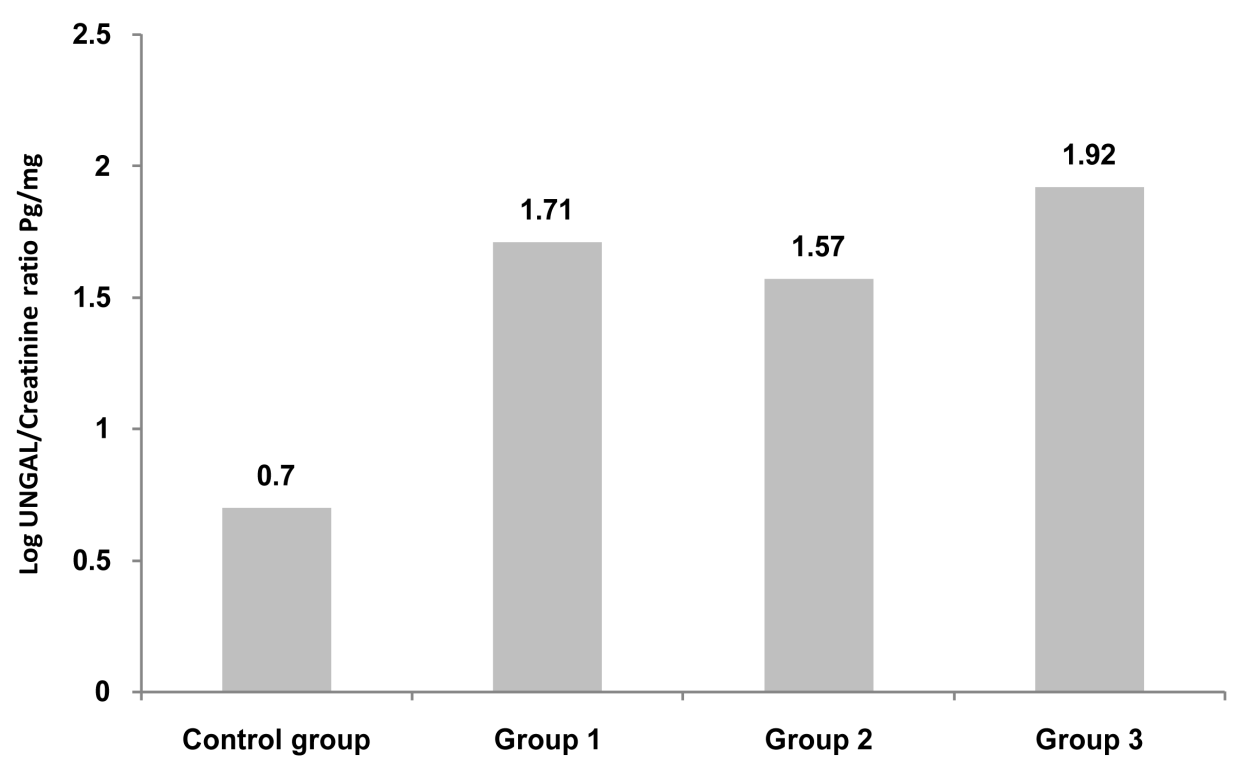

Figure 1. Comparison between studied groups as regard Log UNGAL/Creatinine ratio Pg/mg. Logarithm Urinary Neutrophil Gelatinase Associated lipocaline.
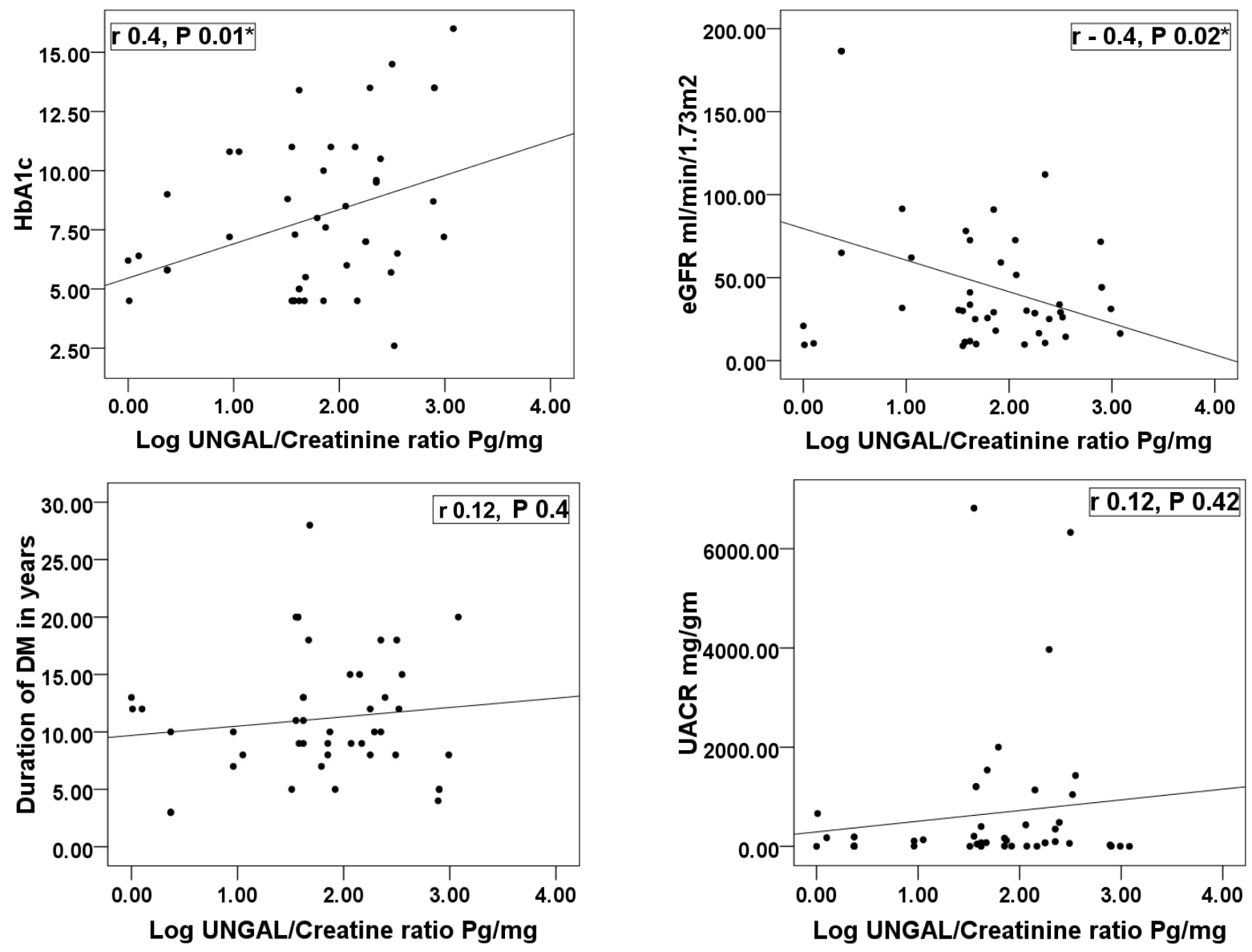

Figure 2. Correlation between log UNGAL/Creatinine ratio with, HbA1c, eGFR ml/min/1.73 $\mathrm{m}^{2}$, duration of diabetes in years and UACR mg/gm. UNGAL; Urinary neutrophil gelatinase associated lipocalin, Log; Logarithm, UACR; Urinary albumin creatinine ratio, eGFR; Estimated glomerular filtration rate, DM; Diabetes Mellitus, "Significant difference. 
Table 2. linear regression analysis to detect the predictors of Log UNGAL/Creatinine ratio.

\begin{tabular}{ccc}
\hline & Log UNGAL/Creatinine ratio Pg/mg & \\
\hline & $\beta$ & $\mathrm{P}$ \\
\hline Duration of DM in years & -0.115 & 0.52 \\
Mean blood pressure & 0.013 & 0.92 \\
HbA1c & 0.411 & $0.003^{*}$ \\
Fasting blood glucose & -0.22 & 0.98 \\
UACR mg/gm & -0.103 & 0.47 \\
Creatinine & 0.15 & 0.94 \\
Urea & -0.022 & 0.98 \\
Urine creatinine & -0.548 & $0.001^{*}$ \\
eGFR ml/min/1.73m & -0.434 & $0.014^{*}$ \\
\hline
\end{tabular}

Log UNGAL/Creatinine: Logarithm urinary neutrophil gelatinase associated lipocaline/Creatinine ratio, DM: Diabetes mellitus, HbA1c: Glycated hemoglobin, UACR: Urinary albumin creatinine ratio, eGFR: Estimated glomerular filtration rate, ${ }^{*}$ Significant difference.

Table 3. AUC, sensitivity and specificity of cut off value of log UNGAL/creatnine ratio.

\begin{tabular}{cccccccc}
\hline AUC & SE & $\begin{array}{c}\text { Asymptomatic } \\
\text { Significance }\end{array}$ & \multicolumn{2}{c}{ Asymptomatic 95\% Confidence Interval } & Sensitivity & Specificity & Cut off point \\
\cline { 4 - 7 } & & Lower bond & Upper bond & & So.4\% & $80 \%$ & 1.035 \\
\hline
\end{tabular}

AUC: Area under the curve, SE: Standard error.

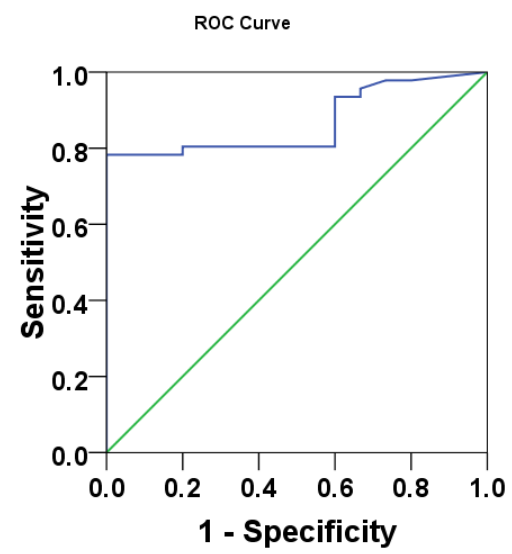

Figure 3. Receiving operation characteristic curve of UNGAL/Creatinine ratio Pg/mg to detect cut off value of raising log UNGAL/Creatinine ratio considering control group as status variable.

di-abetic patients with albuminuria (microalbuminuria and macroalbuminuria) and those without (Normoalbuminuria). Also a control group without diabetes and CKD was compared with all diabetic groups. In our study we found that there was a significant difference among the studied 4 groups (Control, Normoalbuminuria, Microalbuminuria and Macroalbuminuria) regarding log UNGAL/Creatinine ratio while comparison of each 2 groups separately showed that there was significant difference between the control group with each group separately while the diabetic groups did not show significant difference among themselves. It means that UNGAL increased in diabetic patients without albuminuria when compared with control group suggesting that UNGAL as a marker of tubular injury precede the appearance of microalbuminuria as a marker of glomerular injury. In accordance with our results, Bolignano D. et al. who evaluated serum and UNGAL in diabetic patients with albuminuria and found that serum and UNGAL was significantly elevated in diabetic patients in respect to control group, Also like our results he found elevated levels in diabetic patients without early signs of glomerular damage (Normoalbuminuria) [10]. Nauta FL et al. demonstrated that NGAL is 1.5 fold significantly elevated in diabetic patients with normoalbuminuria compared to non-diabetic control group [11]. In an experimental animal study, Miller ML et al. found that urinary biomarkers including UNGAL were elevated in an established rat 
model of diabetic nephropathy and concluded that these biomarkers appeared even before the classical biomarkers of diabetic nephropathy such as albuminuria [30]. Another study by Lacquaniti A. et al. concluded that NGAL increases in patients with type 1 diabetes, even before diagnosis of microalbuminuria representing an early biomarker of normoalbuminuric diabetic nephropathy with a good sensitivity and specificity. NGAL measurement could be useful for the evaluation of early renal involvement in the course of diabetes [31]. Also in children some reports showed that serum and UNGAL were elevated in diabetic children without albuminuria and concluded that Normal-range albuminuria does not exclude diabetic nephropathy defined as increased serum and UNGAL concentration and NGAL measurement can be more sensitive than microalbumin and may become a useful tool for evaluating renal involvement in diabetic children [32]. Some other studies showed inconsistency with our results. Kim S.S. et al. found that UNGAL was elevated in diabetic patients with macroalbuminuria compared to control, normoalbuminuria and microalbuminuria groups, however he did not find any significant difference between control group and normoalbuminuria group [33]. Although Nielsen S.E. et al. found that UNGAL is elevated in diabetic patients with and without albuminuria similar to our results, however in contrast to our results he found that UNGAL increases significantly with increasing albuminuria [34]. Also WU J. et al. was observed that baseline level of urinary NGAL was significantly elevated and correlated with the severity of albuminuria in patients with diabetes and during the follow up, the urinary level of NGAL was observed to be significantly correlated with a rapid decline in the eGFR [35]. In our study log UNGAL/Creatinine ratio showed significant positive correlation with HbA1c and significant negative correlation with eGFR, while it did not show any correlation with UACR and duration of diabetes. Some studies found significant inverse correlation between UNGAL and eGFR like our finding however in contrast to our results they found also significant correlation with albuminuria [10] [36]. Similar to our results Woo et al. found inverse correlation between UNGAL with eGFR while he did not find any correlation with albuminuria [37]. In contrast to our results, Zachwieja J. et al. found correlation between UNGAL and UAE while he did not find any correlation with eGFR and HbA1c [32]. In our study, linear regression analysis demonstrated that independent predictors of log UNGAL/Cr ratio were HbA1c, urine creatinine and eGFR. It means that UNGAL as a marker of tubular damage is highly associated with uncontrolled diabetes and decline of kidney function detected by decreased urinary creatinine and eGFR. We could not find any association between tubular damage detected by log UNGAL/Creatine ratio and glomerular damage detected by microalbuminnria as albuminuria could not predict log UNGAL/Creatinine ratio and also log UNGAL/Creatinine ratio could not predict development of microalbuminuria (Data not shown). So according our data both tubular and glomerular damage occur simultaneously in diabetic patients and we could not decide if the underlying mechanisms are the same or different. We could not find any study determined the independent predictors of urinary NGAL. Most of the studies addressed the value of UNGAL as a predictor of acute kidney injury [38]-[40]. We constructed a ROC curve to detect the cut off value of log UNGAL/Creatinine ratio to determine the normal value of $\log$ UNGAL/Creatinine ratio and it was found $1.035 \mathrm{Pg} / \mathrm{ml}$ with good AUC, sensitivity and specificity. There are some limitations of our work. First the small number of patients, second it is an observational study and we did not address the pathophysiology and underlying mechanisms for tubular injury and if there is an interplay between tubular and glomerular damage, third we did not follow up the patients to see the effect of tight control of diabetes on regression of UNGAL to confirm the role of uncontrolled diabetes on pathogenesis of tubular injury.

\section{Conclusion}

Tubular damage and tubular markers are highly associated with diabetic kidney disease patients. Tubular damage may precede glomerular injury. Tubular markers like UNGAL may be an early marker for detection of kidney involvement in diabetes, and it can be early elevated even before early signs of glomerular injury detected by microalbuminuria. More studies are needed to confirm our results and to detect the underlying mechanisms of tubular injury in diabetic patients.

\section{References}

[1] KDOQI (2012) KDOQI Clinical Practice Guideline for Diabetes and CKD: 2012 Update. American Journal of Kidney Diseases, 60, 850-886. http://dx.doi.org/10.1053/j.ajkd.2012.07.005

[2] Sarnak, M.J., Levey, A.S., Schoolwerth, A.C., Coresh, J., Culleton, B., Hamm, L.L., McCullough, P.A., Kasiske, B.L., 
Kelepouris, E., Klag, M.J., Parfrey, P., Pfeffer, M., Raij, L., Spinosa, D.J. and Wilson, P.W. (2003) Kidney Disease as a Risk Factor for Development of Cardiovascular Disease: A Statement from the American Heart Association Councils on Kidney in Cardiovascular Disease, High Blood Pressure Research, Clinical Cardiology, and Epidemiology and PREVENTION. Hypertension, 42, 1050-1065. http://dx.doi.org/10.1161/01.HYP.0000102971.85504.7c

[3] American Diabetes Association (2004) Position Statement: Nephropathy in Diabetes. Diabetes Care Supplement, 27, 79-83.

[4] Narita, T., Hosoba, M., Kakei, M. and Ito, S. (2006) Increased Urinary Excretions of Immunoglobulin G, Ceruloplasmin, and Transferrin Predict Development of Microalbuminuria in Patients with Type 2 Diabetes. Diabetes Care, 29, 142-146. http://dx.doi.org/10.2337/diacare.29.01.06.dc05-1063

[5] Barratt, J. and Topham, P. (2007) Urine Proteomics: The Present and Future of Measuring Urinary Protein Components in Disease. CMAJ, 177, 361-369. http://dx.doi.org/10.1503/cmaj.061590

[6] Bangstad, H.J., Seljeflot, I., Berg, T.J., and Hanssen, K.F. (2009) Renal Tubulointerstitial Expansion Is Associated with Endothelial Dysfunction and Inflammation in Type 1 Diabetes. Scandinavian Journal of Clinical \& Laboratory Investigation, 69, 138-144. http://dx.doi.org/10.1080/00365510802444080

[7] Thomas, M.C., Burns, W.C. and Cooper, M.E. (2005) Tubular Changes in Early Diabetic Nephropathy. Advances in Chronic Kidney Disease, 12, 177-186. http://dx.doi.org/10.1053/j.ackd.2005.01.008

[8] Haase, M., Bellomo, R., Devarajan, P., Schlattmann, P. and Haase-Fielitz, A. (2009) Accuracy of Neutrophil Gelatinase-Associated Lipocalin (NGAL) in Diagnosis and Prognosis in Acute Kidney Injury: A Systematic Review and Meta-Analysis. American Journal of Kidney Diseases, 54, 1012-1024. http://dx.doi.org/10.1053/j.ajkd.2009.07.020

[9] Peres, L.A., Cunha Júnior, A.D., Schäfer, A.J., Silva, A.L., Gaspar, A.D., Scarpari, D.F., Alves, J.B., Girelli Neto, R. and Oliveira, T.F. (2013) Biomarkers of Acute Kidney Injury. Jornal Brasileiro de Nefrologia, 35, 229-236. http://dx.doi.org/10.5935/0101-2800.20130036

[10] Bolignano, D., Lacquaniti, A., Coppolino, G., Donato, V., Fazio, M.R. and Nicocia, G. (2009) Neutrophil GelatinaseAssociated Lipocalin as an Early Biomarker of Nephropathy in Diabetic Patients. Kidney and Blood Pressure Research, 32, 91-99. http://dx.doi.org/10.1159/000209379

[11] Nauta, F.L., Boertien, W.E., Bakker, S.J., van Goor, H., van Oeveren, W. and de Jong, P.E. (2011) Glomerular and Tubular Damage Markers Are Elevated in Patients with Diabetes. Diabetes Care, 34, 975-981. http://dx.doi.org/10.2337/dc10-1545

[12] Tesch, G.H. and Lim, A.K. (2011) Recent Insights into Diabetic Renal Injury from the db/db Mouse Model of Type 2 Diabetic Nephropathy. American Journal of Physiology. Renal Physiology, 300, 301-310. http://dx.doi.org/10.1152/ajprenal.00607.2010

[13] Stanton, R.C. (2011) Oxidative Stress and Diabetic Kidney Disease. Current Diabetes Reports, 11, 330-336. http://dx.doi.org/10.1007/s11892-011-0196-9

[14] Lim, A.K. and Tesch, G.H. (2012) Inflammation in Diabetic Nephropathy. Mediators of Inflammation, 2012, Article ID: 146154. http://dx.doi.org/10.1155/2012/146154

[15] Paragas, N., Qiu, A., Zhang, Q., Samstein, B., Deng, S.X., Schmidt-Ott, K.M., Viltard, M., Yu, W., Forster, C.S., Gong, G., Liu, Y., Kulkarni, R., Mori, K., Kalandadze, A., Ratner, A.J., Devarajan, P., Landry, D.W., D’Agati, V., Lin, C.S. and J. Barasch, (2011) The NGAL Reporter Mouse Detects the Response of the Kidney to Injury in Real Time. Nature Medicine, 17, 216-222. http://dx.doi.org/10.1038/nm.2290

[16] Ding, H., He, Y., Li, K., Yang, J., Li, X. and Lu, R. (2007) Urinary Neutrophil Gelatinase-Associated Lipocalin (NGAL) Is an Early Biomarker for Renal Tubulointerstitial Injury in IgA Nephropathy. Clinical Immunology, 123, 227-234. http://dx.doi.org/10.1016/j.clim.2007.01.010

[17] Nielsen, S.E., Hansen, H.P., Jensen, B.R., Parving, H.H. and Rossing, P. (2011) Urinary Neutrophil Gelatinase-Associated Lipocalin and Progression of Diabetic Nephropathy in Type 1 Diabetic Patients in a Four-Year Follow-up Study. Nephron Clinical Practice, 118, 130-135. http://dx.doi.org/10.1159/000320615

[18] Levey, A.S., Coresh, J., Greene, T., Stevens, L.A., Zhang, Y.L. and Hendriksen, S. (2006) Using Standardized Serum Creatinine Values in the Modification of Diet in Renal Disease Study Equation for Estimating Glomerular Filtration Rate. Annals of Internal Medicine, 145, 247-254. http://dx.doi.org/10.7326/0003-4819-145-4-200608150-00004

[19] Burtis, E., Santos-Rosa, M., Bienvenu, J. and Whicher, J. (2006) Role of the Clinical Laboratory in Diabetes Mellitus. In: C. Bruit and E. Ashwood, Eds., Tietz Textbook of Clinical Chemistry Chapter 25, 4th Edition, Mosby, St. Louis, 837-903.

[20] Larsen, K. (1972) Creatinine Assay by a reaCtion-Kinetic Principle. Clinica Chimica Acta, 41, 209-217. http://dx.doi.org/10.1016/0009-8981(72)90513-X

[21] Talke, H. and Schubert, G.E. (1965) Enzymatic Determination of Urea Using the Coupled Urease-GLDH Enzyme System. Mediators of Inflammation, 43, 174-176. http://dx.doi.org/10.1007/BF01484513 
[22] Gonen, B. and Rubenstein, A.H. (1978) Determination of Glycohemoglobin. Diabetologia, 15, 1-5. http://dx.doi.org/10.1007/BF01219319

[23] Rowe, D.J.F., Dawnay, A. and Watts, G.F. (1990) Microalbuminuria in Diabetes Mellitus: Review and Recommendations for the Measurement of Albumin in Urine. Annals of Clinical Biochemistry, 27, 297-312. http://dx.doi.org/10.1177/000456329002700404

[24] Rodby, R.A., Rohde, R.D., Sharon, Z., Pohl, M.A., Bain, R.P. and Lewis, E.J. (1995) The Urine Protein to Creatinine Ratio as a Predictor of 24-Hour Urine Protein Excretion in Type 1 Diabetic Patients with Nephropathy. The Collaborative Study Group. American Journal of Kidney Diseases, 26, 904-909. http://dx.doi.org/10.1016/0272-6386(95)90054-3

[25] Brunner, H.I., Mueller, M., Rutherford, C., Passo, M.H., Witte, D. and Grom, A. (2006) Urinary Neutrophil Gelatinase-Associated Lipocalin as a Biomarker of Nephritis in Childhood-Onset Systemic Lupus Erythematosus. Arthritis \& Rheumatology, 54, 2577-2584. http://dx.doi.org/10.1002/art.22008

[26] Gray, S.P. and Cooper, M.E. (2011) Diabetic Nephropathy in 2010: Alleviating the Burden of Diabetic Nephropathy. Nature Reviews Nephrology, 7, 71-73. http://dx.doi.org/10.1038/nrneph.2010.176

[27] Mishra, J., Dent, C., Tarabishi, R., Mitsnefes, M.M., Ma, Q., Kelly, C., Ruff, C., S.M., Zahedi, K., Shao, M., Bean, J., Mori, K., Barasch, J. and Devarajan, P. (2005) Neutrophil Gelatinase-Associated Lipocalin (NGAL) as a Biomarker for Acute Renal Injury after Cardiac Surgery. Lancet, 8, 1231-1238. http://dx.doi.org/10.1016/S0140-6736(05)74811-X

[28] Bolignano, D., Coppolino, G., Campo, S., Aloisi, C., Nicocia, G., Frisina, N. and Buemi, M. (2008) Urinary Neutrophil Gelatinase-Associated Lipocalin (NGAL) Is Associated with Severity of Renal Disease in Proteinuric Patients. Nephrology Dialysis Transplantation, 23, 414-416. http://dx.doi.org/10.1093/ndt/gfm541

[29] Bolignano, D., Lacquaniti, A., Coppolino, G., Donato, V., Campo, S., Fazio, M.R., Nicocia, G. and Buemi, M. (2009) Neutrophil Gelatinase-Associated Lipocalin (NGAL) and Progression of Chronic Kidney Disease. Clinical Journal of the American Society of Nephrology, 4, 337-344. http://dx.doi.org/10.2215/CJN.03530708

[30] Alter, M.L., Kretschmer, A., Von Websky, K., Tsuprykov, O., Reichetzeder, C., Simon, A., Stasch, J.P. and Hocher, B. (2012) Early Urinary and Plasma Biomarkers for Experimental Diabetic Nephropathy. Clinical Laboratory, 58, 659671.

[31] Lacquaniti, A., Donato, V., Pintaudi, B., Di Vieste, G., Chirico, V., Buemi, A., Di Benedetto, A., Arena, A. and Buemi, M. (2013) “Normoalbuminuric” Diabetic Nephropathy: Tubular Damage and NGA. Acta Diabetologica, 50, 935-942. http://dx.doi.org/10.1007/s00592-013-0485-7

[32] Zachwieja, J., Soltysiak, J., Fichna, P., Lipkowska, K., Stankiewicz, W., Skowronska, B., Kroll, P. and Lewandowska-Stachowiak, M. (2010) Normal-Range Albuminuria Does Not Exclude Nephropathy in Diabetic Children. Pediatric Nephrology, 25, 1445-1451. http://dx.doi.org/10.1007/s00467-010-1443-Z

[33] Kim, S.S., Song, S.H., Kim, J.J., Yang, J.Y., Lee, J.G., Kwak, I.S. and Kim, Y.K. (2012) Clinical Implication of Urinary Tubular Markers in the Early Stage of Nephropathy with Type 2 Diabetic Patients. Diabetes Research and Clinical Practice, 97, 251-257. http://dx.doi.org/10.1016/j.diabres.2012.02.019

[34] Nielsen, S.E., Schjoedt, K.J., Astrup, A.S., Tarnow, L., Lajer, M., Hansen, P.R., Parving, H.H. and Rossing, P. (2010) Neutrophil Gelatinase-Associated Lipocalin (NGAL) and Kidney Injury Molecule 1 (KIM1) in Patients with Diabetic Nephropathy: A Cross-Sectional Study and the Effects of Lisinopril. Diabetic Medicine, 27, 1144-1150. http://dx.doi.org/10.1111/j.1464-5491.2010.03083.x

[35] Wu, J., Ding, Y., Zhu, C., Shao, X., Xie, X., Lu, K. and Wang, R. (2013) Urinary TNF- $\alpha$ and NGAL Are Correlated with the Progression of Nephropathy in Patients with Type 2 Diabetes. Experimental and Therapeutic Medicine, 6, 1482-1488.

[36] Fu, W.J., Xiong, S.L., Fang, Y.G., Wen, S., Chen, M.L., Deng, R.T., Zheng, L., Wang, S.B., Pen, L.F. and Wang, Q. (2012) Urinary Tubular Biomarkers in Short-Term Type 2 Diabetes Mellitus Patients: A Cross-Sectional Study. Endocrine, 41, 82-88.

[37] Woo, K.S., Choi, J.L., Kim, B.R., Kim, J.E., An, W.S. and Han, J.Y. (2012) Urinary Neutrophil Gelatinase-Associated Lipocalin Levels in Comparison with Glomerular Filtration Rate for Evaluation of Renal Function in Patients with Diabetic Chronic Kidney Disease. Diabetes \& Metabolism Journal, 36, 307-313. http://dx.doi.org/10.4093/dmj.2012.36.4.307

[38] Liebetrau, C., Dörr, O., Baumgarten, H., Gaede, L., Szardien, S., Blumenstein, J., Rolf, A., Möllmann, H., Hamm, C., Walther, T., Nef, H. and Weber, M. (2013) Neutrophil Gelatinase-Associated Lipocalin (NGAL) for the Early Detection of Cardiac Surgery Associated Acute Kidney Injury. Scandinavian Journal of Clinical \& Laboratory Investigation, 73, 392-399. http://dx.doi.org/10.3109/00365513.2013.787149

[39] Makris, K., Markou, N., Evodia, E., Dimopoulou, E., Drakopoulos, I., Ntetsika, K., Rizos, D., Baltopoulos, G. and Haliassos, A. (2009) Urinary Neutrophil Gelatinase-Associated Lipocalin (NGAL) as an Early Marker of Acute Kidney 
Injury in Critically Ill Multiple Trauma Patients. Clinical Chemistry and Laboratory Medicine, 47, 79-82. http://dx.doi.org/10.1515/CCLM.2009.004

[40] Wagener, G., Gubitosa, G., Wang, S., Borregaard, N., Kim, M. and Lee, H.T. (2008) Urinary Neutrophil GelatinaseAssociated Lipocalin and Acute Kidney Injury after Cardiac Surgery. American Journal of Kidney Diseases, 52, 425433. http://dx.doi.org/10.1053/j.ajkd.2008.05.018 